\title{
THE IMPACT OF CLONALITY ON PARASITE POPULATION GENETIC STRUCTURE
}

\author{
PRUGNOLLE F.* \& DE MEEÛS T.*
}

\section{Summary:}

In this paper, we briefly review the consequences of clonal reproduction on the apportionment of genetic diversity in parasite populations. We distinguish three kinds of parasite life-cycle where clonal reproduction occurs. The consequences of this mode of reproduction for the different kinds of parasite life-cycles are described. We here particularly focus on clonal diploids.

KEY WORDS : clonality, asexual reproduction, population structure, parasite.

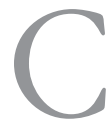
lonality or asexual reproduction corresponds to the production of new individuals genetically identical to the ancestor at all loci in the genome, except at those sites that have experienced somatic mutations (De Meeûs et al., 2007b).

Many parasite species reproduce clonally, at least during one stage of their life-cycle. Depending on their complexity, we may roughly distinguish three kinds of parasite life-cycles (De Meeûs et al., 2007b). The first kind is the life-cycle with only one (I) round of clonality (or LC-I). Parasites that display this life cycle necessarily alternate one phase of sexual reproduction and one phase of asexual reproduction. This life-cycle is typical of trematode parasites (e.g. flukes) as well as of some cestodes (such as Echinoccocus) (Prugnolle et al., 2005a; Prugnolle et al., 2005b). The second kind is the life-cycle with several rounds of asexuality (LC-S). Parasites with this life-cycle also alternate phases of sexual and asexual reproduction, but asexual reproduction may be maintained for several generations before one event of sexual reproduction occurs. LC-S is typical of many fungi and most Sporozoa such as the agents of malaria (Plasmodium sp.). The last kind of life-cycle is what we call the acyclic life-cycle (A-LC) (Balloux et al., 2003). In this case, the life-cycle is not defined by a regular pattern of alternation of sexual or asexual reproduction. Individuals in a population may be issued from either sexual or asexual reproduction. The fre-

\footnotetext{
* Laboratoire Genetics and Evolution of Infectious Disease (GEMI), UMR CNRS-IRD 2724, IRD Montpellier, 911, avenue Agropolis, BP 64501, 34394 Montpellier Cedex 5, France.

Correspondence: Franck Prugnolle.

Tel.: +33 (0)467416310 - Fax: +33 (0)467416299.

E-mail: prugnoll@mpl.ird.fr
}

quency of sexually - or asexually - produced individuals may vary widely and be dominated by either mode of reproduction. This later kind of life-cycle is present in several groups of parasites such as in imperfect fungi (e.g. Candida albicans), in Parabasalia (Trichomonas vaginalis), Metamonadina (Giardia lamblia) and in parasitic amoebas and kinetoplastid parasites (Leishmania, Trypanozoma).

These different life-cycles may have different consequences on the population genetic structure of the concerned parasites. We here make a brief review of the consequences of clonality on the apportionment of genetic variability at different levels, both within and between populations. For the purpose of this review, we will mostly focus our description on clonal diploids.

\section{CLONALITY AND DEVIATION FROM HARDY-WEINBERG EXPECTATIONS}

Since the pioneering works of Hardy \& Weinberg, it is well known that, in a sexually reproducing population of diploid individuals, when gametes meet randomly (panmixia) to form zygotes, the expected genotypic distribution of two alleles (A and a) of frequency $p$ and $q=(1-p)$ in the population has the form $p^{2}$, $2 p q$ and $q^{2} . p^{2}$ and $q^{2}$ represent the expected frequency of homozygous individuals (AA and aa) in the population while $2 p q$ is the expected frequency of individuals that are heterozygous (Aa) (e.g. De Meeûs et al., 2007a). This result is known as the Hardy-Weinberg equilibrium $(\mathrm{H}-\mathrm{W})$. Clonality tends to modify this expected distribution of genotypes.

Deviation from Hardy-Weinberg expectations is generally measured using the statistics $F_{\text {IS }}$ introduced by Wright (1951). This statistics simply measures, within population, the difference between the proportion of observed heterozygotes and the expected proportion of heterozygotes if parents had reproduced randomly. A null $F_{\mathrm{IS}}$ is indicative of panmixia. A positive $F_{\mathrm{IS}}$ means that there is a deficit of heterozygous individuals compared to $\mathrm{H}-\mathrm{W}$ expectations while a negative $F_{\mathrm{IS}}$ is indicative of an excess of heterozygous individuals. 
For parasites that alternate sexual and asexual reproduction (i.e. LC-S and LC-I), if clonality occurs at a diploid stage, the resulting population will show, in average, a smaller $F_{\mathrm{IS}}$ than the parent population (Prugnolle et al., 2005a; Prugnolle et al., 2005b). This is linked to the fact that clonal reproduction tends to decrease the average expected heterozygosity within populations without affecting, in average, the observed heterozygosity. To illustrate this phenomenon, let's consider an extreme example. The population before clonal reproduction (the parent population) displays a null $F_{\mathrm{IS}}$, that is, no deviation from $\mathrm{H}-\mathrm{W}$ expectations. Let's consider now that individuals reproduce clonally and that the resulting population is only made of one final diploid genotype (all resulting diploid individuals have the same genotype at all loci). This may occur in two distinct situations: 1) in the case of parasite with LC-I if the variance in the reproductive success of clones is maximal (only one individual reproduce) or 2) in the case of LC-S if the population is of finite size and the number of clonal generations sufficient to allow, by drift, the fixation of one diploid genotype. In both cases, a fraction of the loci will be homozygous for the same allele in all parasites of the resulting population and $F_{\text {IS }}$ will be undetermined, whereas the remaining loci will be heterozygous in all individuals with an associated $F_{\text {IS }}$ of -1 . The expected $F_{\text {IS }}$ overall loci will thus only be determined by the $F_{\text {IS }}$ at heterozygote loci and will be equal to -1 , a smaller value than the one observed in the parent population where $F_{\text {IS }}$ was equal to 0 . Obviously, this is an extreme example. If the variance in the reproductive success of clones is lower or if clonal genetic drift is not strong enough to lead to the fixation of only one clonal genotype, $F_{\text {IS }}$ will be higher than -1 but still lower than the one observed in the parent population (Prugnolle et al., 2005a; Prugnolle et al., 2005b).

Note that if clonality occurs at a haploid stage, for instance at the gametic stage (as it is the case in Plasmodium sp.), just before sexual reproduction and thus just before the fusion of gametes, it will have no direct effects on the $F_{\text {IS }}$ of the resulting zygotes. In that case, the only thing that may generate a deviation from panmixia in zygotes is whether gametes do not fusion randomly regarding their allelic frequencies.

For acyclic life-cycle parasites (A-LC) with a rate of clonality of 1 (all individuals in the population results from clonal reproduction), clones accumulate heterozygosity over time through mutation events at each locus. This leads to extremely high heterozygote excesses in the populations (low negative $F_{\text {IS }}$ values) (Balloux et al., 2003). In finite populations and without other forces such as migration, each locus will eventually become fixed at an heterozygote state, thus leading to a $F_{\text {IS }}$ of -1 (De Meeûs \& Balloux, 2005; De Meeûs et al., 2006), as explained in the example presented above. As long as the population does not go extinct, there will be at least two alleles present. Note that whenever there is a small amount of sexual reproduction the $F_{\text {IS }}$ rapidly converge towards the one expected under panmixia that is 0 (Balloux et al., 2003).

\section{CLONALITY AND LINKAGE DISEQUILIBRIUM}

I inkage equilibrium is the multilocus equivalent of the $\mathrm{H}-\mathrm{W}$ equilibrium. When gametes meet at random in a population, alleles at different unlinked loci in the genome should be randomly associated. The diversity of multilocus genotype should only be determined by the frequency of the different alleles at each locus. In this case, we say that loci are in linkage equilibrium. On the opposite, when alleles at different loci are not randomly associated regarding their allelic frequencies, we say that these loci are in linkage disequilibrium.

Because clonality is a form of reproduction characterised by an absence of recombination, it tends to produce linkage disequilibrium whatever the life-cycle considered. In the case of A-LC parasites, linkage disequilibrium progressively increases with the rate of clonal reproduction occurring within population. As a consequence, clonality reduces the multilocus genetic diversity (the number of different multilocus genotypes maintained in the population), despite increasing the number of alleles found at each individual locus (De Meeûs \& Balloux, 2004).

\section{CLONALITY AND POPULATION GENETIC DIFFERENTIATION}

$\mathrm{P}$ opulations are genetically differentiated when they present different allelic frequencies. Population differentiation is generally measured thanks to the $F$-statistics $F_{\mathrm{ST}}$ introduced by S. Wright (Wright, 1951). This statistic simply measures the difference between the average expected heterozygosity (or more appropriately genetic diversity) within populations and the expected heterozygosity computed overall populations. Clonal reproduction may affect population differentiation in different ways.

In the case of parasites with LC-I and LC-S, the clonal diploid reproduction will tend to increase the genetic differentiation among populations compared to the parent population. This is due to the fact that, as previously explained, clonal reproduction tends to decrease the average expected heterozygosity within populations but does not change, in average, the expected heterozygosity overall populations. 
In the case of A-LC parasites, populations tend to show lower genetic differentiation than strict sexual organisms, everything else being equal. For instance, in an infinite island model with a migration rate of 0 , the expected $F_{\mathrm{ST}}$ measured between populations is 0.5 instead of 1 in the panmictic case, because two alleles are maintained in each individual within each population (the value 1 is only possible if each population is fixed for one or another allele).

\section{CONCLUSION}

C lonal reproduction affects the apportionment of genetic variation at different levels both within and between populations and leave clear genetic signatures. As mentioned above, the impact of clonality may slightly vary from one life-cycle to another, thus leading to different patterns of genetic structure at individual and population levels. Such knowledge is not purely academic as the use of variable molecular markers can provide very useful information on the population biology of the organism under scrutiny, in particular pathogenic organisms (molecular epidemiology) (Tibayrenc, 1999).

\section{ACKNOWLEDGEMENTS}

The authors are financed by the CNRS and the IRD.

\section{REFERENCES}

Balloux F., Lehmann L. \& De MeEûs T. The population genetics of clonal and partially clonal diploids. Genetics, 2003, 164, 1635-1644.

De Meeûs T. \& Balloux F. Clonal reproduction and linkage disequilibrium in diploids: a simulation study. Infection Genetics and Evolution, 2004, 4, 345-351.

De Meeûs T. \& Balloux F. F-statistics of clonal diploids structured in numerous demes. Molecular Ecology, 2005, 14, 2695-2702.

De Meeûs T., Lehmann L. \& Balloux F. Molecular epidemiology of clonal diploids: A quick overview and a short DIY (do it yourself) notice. Infection Genetics and Evolution, 2006, 6, 163-170.

De Meeûs T., McCoy K. D., Prugnolle F., Chevillon C., Durand P., Hurtrez-Boussès S. \& Renaud F. Population genetics and molecular epidemiology or how to "débusquer la bête". Infection Genetics and Evolution, 2007a, 7, 308-332.

De Meeûs T., Prugnolle F. \& Agnew P. Asexual reproduction: Genetics and evolutionary aspects. Cellular and Molecular Life Sciences, 2007b, 64, 1355-1372.

Prugnolle F., Liu H., De Meeûs T. \& Balloux F. Population genetics of complex life-cycle parasites: an illustration with trematodes. International Journal for Parasitology, 2005a, 35, 255-263.

Prugnolle F., Roze D., Théron A. \& De Meeûs T. F-statistics under alternation of sexual and asexual reproduction: a model and data from schistosomes (platyhelminth parasites). Molecular Ecology, 2005b, 14, 1355-1365.

Tibayrenc M. Toward an integrated genetic epidemiology of parasitic protozoa and other pathogens. Annual Review of Genetics, 1999, 33, 449-477.

Wright S. The genetical structure of populations. Annals of Eugenics, 1951, 15, 323-354. 